Document downloaded from:

http://hdl.handle.net/10251/65782

This paper must be cited as:

Poza-Lujan, J.; Calafate, CT.; Posadas-Yagüe, J.; Cano, J. (2016). Assessing the impact of continuous evaluation strategies: tradeoff between student performance and instructor effort. IEEE Transactions on Education. 59(1):17-23. doi:10.1109/TE.2015.2418740.

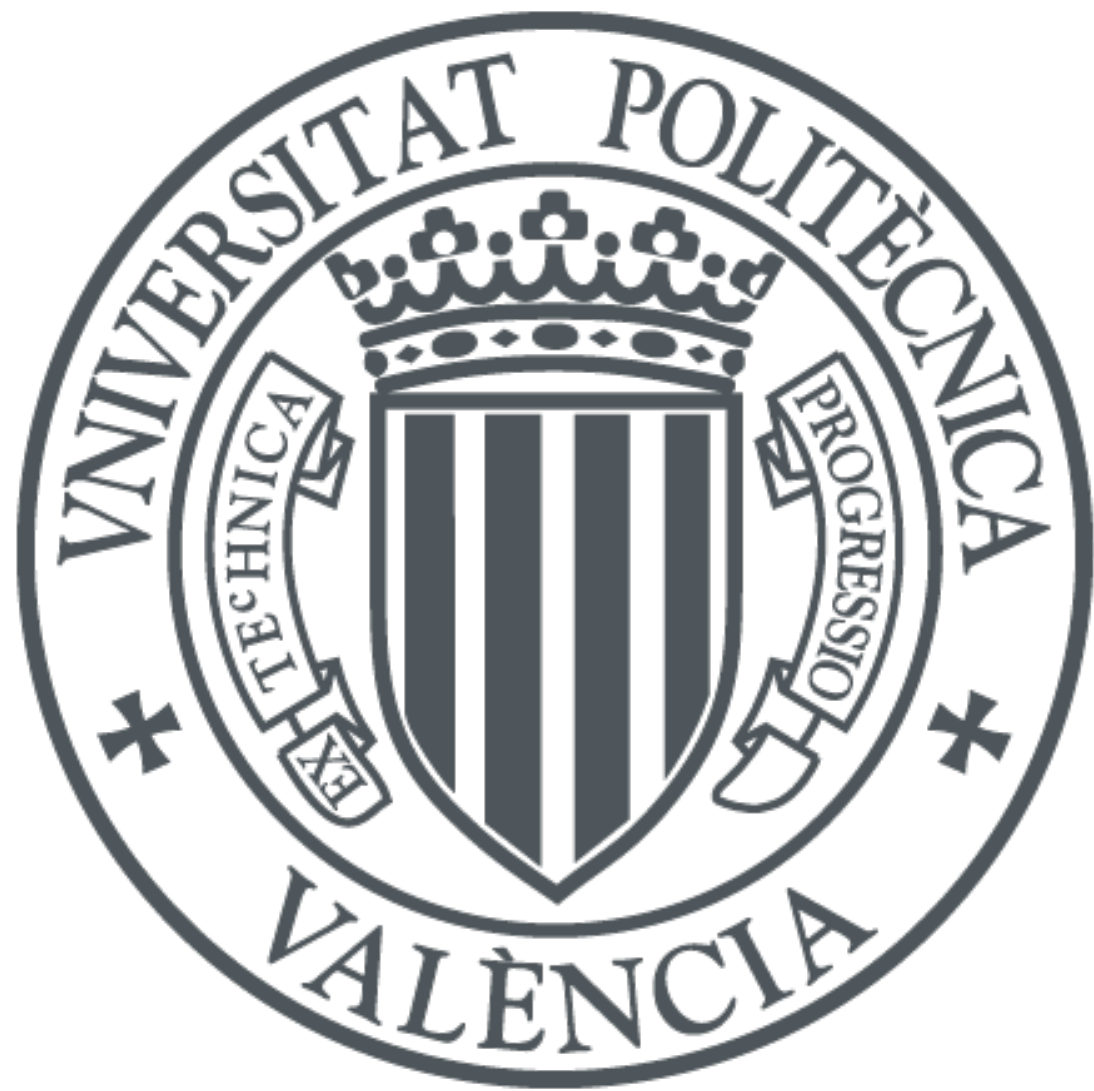

The final publication is available at

http://dx.doi.org/10.1109/TE.2015.2418740

Copyright Institute of Electrical and Electronics Engineers (IEEE)

Additional Information

(C) 2016 IEEE. Personal use of this material is permitted. Permission from IEEE must be obtained for all other uses, in any current or future media, including reprinting/republishing this material for advertising or promotional purposes, creating new collective works, for resale or redistribution to servers or lists, or reuse of any copyrighted component of this work in other works 


\title{
Assessing the Impact of Continuous Evaluation Strategies: Tradeoff Between Student Performance and Instructor Effort
}

\author{
Jose-Luis Poza-Lujan, Member, IEEE, Carlos T. Calafate, Juan-Luis Posadas-Yagüe and Juan-Carlos \\ Cano
}

\begin{abstract}
Current opinion on undergraduate studies has led to a reformulation of teaching methodologies to base them not just on learning, but also on skills and competencies. In this approach, the teaching/learning process should accomplish both knowledge assimilation and skill development. Previous works demonstrated that a strategy that uses continuous evaluation is able to meet both objectives. However, those studies did not evaluate and quantify the additional effort required to implement such strategies. This paper evaluates the additional instructor effort required when implementing continuous evaluation in a first-year Computer Fundamentals course on the Computer Engineering degree program at the Technical University of Valencia, Spain. The experiment quantifies how instructor workload increases under different continuous evaluation strategies, and how this affects the overall student grade. Both the "standard" continuous evaluation method and the intensive continuous evaluation method are analyzed; the latter being a proposal that builds upon the standard method by increasing the number of tests and examinations. The results obtained reveal that continuous evaluation improves student grades, but that intensive continuous evaluation is liable to generate an excessive instructor overload without having a significant impact on student scores.
\end{abstract}

Index Terms-Continuous assessment, formative assessment, instructor workload, student performance.

\section{INTRODUCTION AND MOTIVATION}

$\mathrm{T}$ HE process of European universities adapting to the European Higher Education Area (EHEA) has introduced significant changes to teaching methodologies and the evaluation methods [1]. Classical evaluation, as applied in the university degree programs for decades past, consisted of a single exam at the end of the academic semester/year, with its result defining the course grade for each student. In contrast, new methodologies recently implemented in university

Manuscript received October 09, 2014; revised January 13, 2015; accepted March 15, 2015. Authors want to thank the support of the Department of Computing Engineering (DISCA) and the School of Informatics (ETSINF) of the Universitat Politècnica de València (UPV). Special thanks to teachers and students involved in this study.

All authors contributed equally to this work. The authors are with the Department of Computer Engineering (DISCA) of the School of Informatics (ETSINF), The Universitat Politècnica de València (UPV), 46022 Valencia, Spain (e-mail: jopolu@disca.upv.es, calafate@disca.upv.es, jposadas@disca.upv.es, jucano@disca.upv.es) degrees make the student more explicitly and continuously involved by having them perform work both inside and outside of class; an evaluation system that can track student achievements throughout the course is thus required [2]. These new evaluation methodologies both encourage students to keep up to date with their studies so as to gradually acquire the competencies covered in the course, and provide feedback to instructors about the effectiveness of the work being done, so they can employ alternative methodologies as necessary.

The various European higher education stakeholders involved in the EHEA convergence process are aware of its implications, which include degree structure, credit definition, and the active role of students. But, as stated in [1], other aspects of the methodology, such as student assessment, can complicate its practical application, and can require reflection, adaptation, and improvement.

Several previous studies have addressed these issues. In [3] the authors present an evaluation methodology that allows the student to choose between a final exam or a continuous assessment based on the average of three exams. This method, which applies to both theory and laboratory sessions, raises an interesting reflection about the cost of this for instructors; no empirical data were provided on this aspect.

In [4] the author presents an extensive review study that analyzes the changes introduced by competency-based studies on the design of evaluation, paying particular attention to the type of assessment activity. The results of this study illustrate the convenience of using continuous assessment. The ultimate goal is to provide instructors with the tools required to implement the new evaluation paradigm in their courses. Based on this study, the authors of [5] discuss the feasibility of continuous assessment in universities, presenting a continuous assessment model based on competencies, and particularizing the model to both face-to-face and virtual environments.

Other work, [6], points out that continuous assessment provides a clear view of the progress and learning difficulties of students, offering the instructor all the information required to dynamically adapt the learning process. This work elicited the opinions of one thousand second-year undergraduate students, and concluded that continuous evaluation strategies allow students to remember the course contents better, without the stress produced by the final (exam-based) evaluation.

Focusing on engineering degrees, Christofourou et al. [7] 
TABLE I

COURSE UNITS AND HOURS SPENT PER UNIT

\begin{tabular}{lll}
\hline \multicolumn{1}{c}{ Unit } & $\begin{array}{c}\text { Class and } \\
\text { seminar hours }\end{array}$ & Lab hours \\
\hline 1. Introduction to computers & 3.0 & 0.0 \\
2. Principles of digital design & 9.0 & 3.0 \\
3. Basic combinational blocks & 4.5 & 1.5 \\
4. Sequential circuits & 3.0 & 1.5 \\
5. Design and analysis of synchronous & 7.5 & 3.0 \\
sequential systems & & \\
6. Representation of information & 7.5 & 1.5 \\
7. Introduction to the assembly language & 10.5 & 4.5 \\
\hline \hline
\end{tabular}

presented a methodology for continuous assessment that pursues the accreditation of studies based on feedback and continuous improvement of the learning process. Specifically, a model based on continuous assessment is designed that evaluates the students' learning process while simultaneously identifying possible drawbacks associated with that process. The authors show that the model must be accompanied by adequate tools that allow quantitative measurement of the learning process in terms of both competencies and skills acquired by students. They also note the advisability of using continuous assessment to align student outcomes with the ABET engineering criteria educational objectives [8].

In terms of instructor workload, the authors of [2] conclude that $93.2 \%$ of teachers feel that EHEA implementation has caused their workload to increase. The study did not evaluate time invested in implementing continuous assessment, however, so to measure this time would certainly be valuable effort. Instructor workload is measured in [3] and [9]; both studies conclude that the time invested on continuous assessment is more than the time invested in final assessment (although they did not perform both assessment types simultaneously). In [3] the instructor workload was increased by up to $90 \%$, and in [9] by more than $100 \%$. Previous work suggests that it is worth studying in detail the relationship between continuous assessment, student results, and additional instructor workload.

All these studies emphasize the importance of continuous assessment in the light of the new European higher education framework, discussing its applicability to competence-based learning models, and highlighting the need for new tools to validate these models. However, none presents a quantitative analysis of the effort involved in such changes, or highlights the need to seek a compromise between instructor effort and student results. The work reported in this paper attempts to quantify the effort made by instructors when implementing continuous evaluation in a course, measuring the impact of that effort on improvements in student scores.

Two methodologies for continuous evaluation are analyzed: the "standard" continuous evaluation method, that consists of correcting exercises related to the contents of each unit in the classroom, and a proposed methodology named "intensive continuous evaluation", which builds upon this method by adding a written test at the end of each unit.

The study was conducted during four consecutive academic years (2009-10, 2010-11, 2011-12, and 2012-13) in Computer
Fundamentals (FCO), a compulsory first-year course in the Computer Engineering degree program at the Technical University of Valencia, Spain. The first of these academic years (2009-10) was used as a control reference for comparison. In that particular academic year, the only evaluation made was the final exam. During the next three course offerings (2010-11, 2011-12 and 2012-13), continuous evaluation was implemented in ten control groups, and intensive continuous evaluation was implemented during the 2011-12 and 2012-13 academic years for one experimental group.

Metrics proposed by the authors to measure the instructor load associated with continuous evaluation are presented below, as are the results of these measurements and an analysis of student performance. The objective was to understand the trade-off between instructor effort and student performance, when implementing the proposed methodology. The results show that instructor academic load increases between $53 \%$ and $80 \%$ when switching to intensive continuous evaluation, and student scores increase by about $14 \%$. So, achieving an increase of one percentage point in student performance increases academic load by five percentage points.

The rest of the paper is organized as follows. The course description and the proposed methodology are described in Section II. The results obtained are examined and discussed in Section III, addressing issues such as the cost of continuous evaluation in terms of efficiency, and comparing instructor effort to student performance. Conclusions and future work are then presented in Section IV.

\section{METHODOLOGY AND FRAMEWORK}

This section places the study into its theoretical context, and describes the proposed methodology. It begins by presenting the course description and the available evaluation methodologies, then presents and analyzes the proposed methodology, and considers the instructor's tasks in the continuous evaluation process.

\section{A. Course Description}

The Computer Fundamentals (FCO) course was first offered during the 2010-2011 academic year as an evolution of a course with the same name, following the recommendations of the European Higher Education Area (EHEA) [10], which in particular recommend the use of more diversified methods of evaluation that are able to reflect students' results not in examinations, but also in lab activities, presentations, coursework, and so on.

Taking these issues into account, the FCO course was allocated 6 ECTS credits, distributed between theoretical sessions (25\%), seminars (50\%), and laboratory sessions (25\%). The course contents are organized into seven units, Table I. Currently, the course has 11 lecture/seminar sections each year, with an average of 50 students grouped in each section. Each lecture/seminar section is divided into two groups for practical laboratory work, to give some 25 students in each lab session, so the teacher can offer them individual 
attention.

\section{B. Evaluation Methodologies}

There is some consensus on classifying types of pedagogic evaluation as a function of time [11], as: (i) diagnostic, commonly called pre-evaluation, (ii) formative, usually known as continuous, and (iii) summative, usually known as final evaluation [12]. Pre-assessment is understood as an evaluation task performed before the formative process starts, and is designed to assess the students' level before facing that process. Continuous evaluation is carried out throughout the whole process, to provide feedback to both students and instructors about how the teaching/learning process is progressing. Lastly, final evaluation is made at the end of the formative process, to determine the level achieved by students.

Both evaluation methods, continuous and final, are useful to the learning process, and are thus not mutually exclusive. In [13] a good comparison of the usefulness of both evaluation methods is given: "When the cook tastes the soup, that's formative, when the guests taste the soup, that's summative". Hence, it is interesting to experiment simultaneously with both types of evaluation. Continuous evaluation provides feedback to both teachers and students, allowing the former to take some strategic decisions like changing the type of exercises, while also allowing the latter to regulate their study time. The final evaluation allows teachers (or employers) to know the level achieved at the end of the teaching-learning process.

Within the EHEA, the term "continuous evaluation" refers to the process of assessing the evolution of a student throughout the whole academic year, instead of relying solely on the result of a final test to determine knowledge and skill acquisition levels. Nevertheless, as with final evaluation, ongoing evaluation requires clear definition of how students will be assessed and preparation of the various evaluation tests taken throughout the course.

According to the Academic Standards and Student Assessment regulations at the Technical University of Valencia [14], an evaluation test (or assessment act) is defined as "any test, exercise or examination that influences the final score" with a maximum duration of four hours. For the instructor, the time overhead for each evaluation test goes well beyond the defined test schedule. In fact, the tasks associated with every evaluation test typically start long before, and finish long after, the test. Fig. 1 shows these various instructor tasks: preparation, implementation, correction, and finally the evaluation review.

The preparation phase includes the proposing/selecting

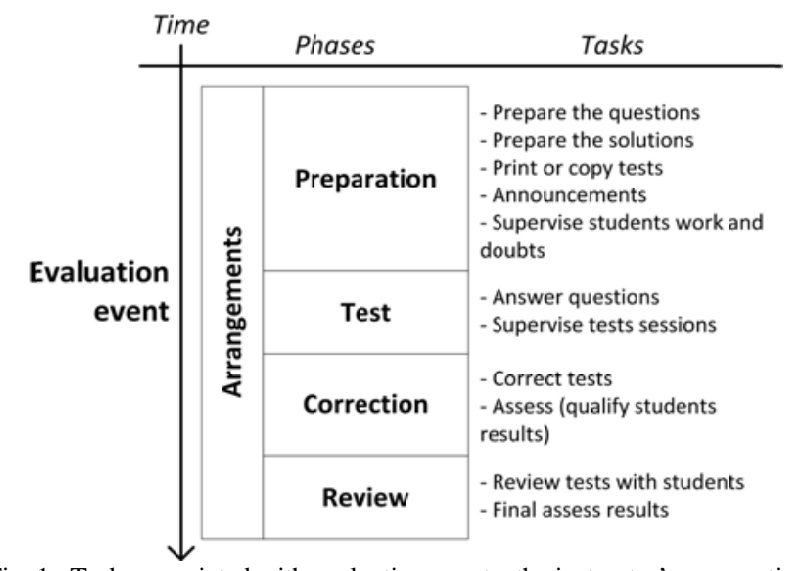

Fig. 1. Tasks associated with evaluation events: the instructor's perspective.

questions (which can be quite complex if a consensus must be reached between all instructors involved) and preparing their test solutions. Additional issues of organizing of the event, and preparing for potential problems encountered by students during the event, are also part of the preparation. The test phase is the evaluation event itself, that is, the period during which students solve the test. The main task of the correction phase is the marking of every student's test. Depending of the type of test this phase may impose a large teacher workload; for example, a test based on multiple-choice answers can be easily corrected, but an open-answer test is much more time consuming. Finally, for all phases, the management tasks (particularly instructor meetings) must be considered.

\section{Proposed Methodology}

The proposed "intensive" continuous evaluation methodology builds upon the "standard" continuous evaluation methodology by adding a written test at the end of each unit. The students under analysis were organized into ten control groups, receiving "standard" continuous evaluation, and one experimental group, receiving intensive continuous evaluation. In addition, both the control and the experimental groups performed a common final evaluation, which consisted of an exam at the end of each semester, so the exam scores could be used to compare the effectiveness of the intensive continuous evaluation method against standard continuous evaluation in terms of learning outcome measured, and instructor load. The experimental group was selected at random from the eleven sections taking the course. The remaining sections were considered as control groups. Therefore, students were not specially selected to populate the experiment group; in fact those students did not know that a different methodology had been adopted. The same section teachers taught all course offerings. 


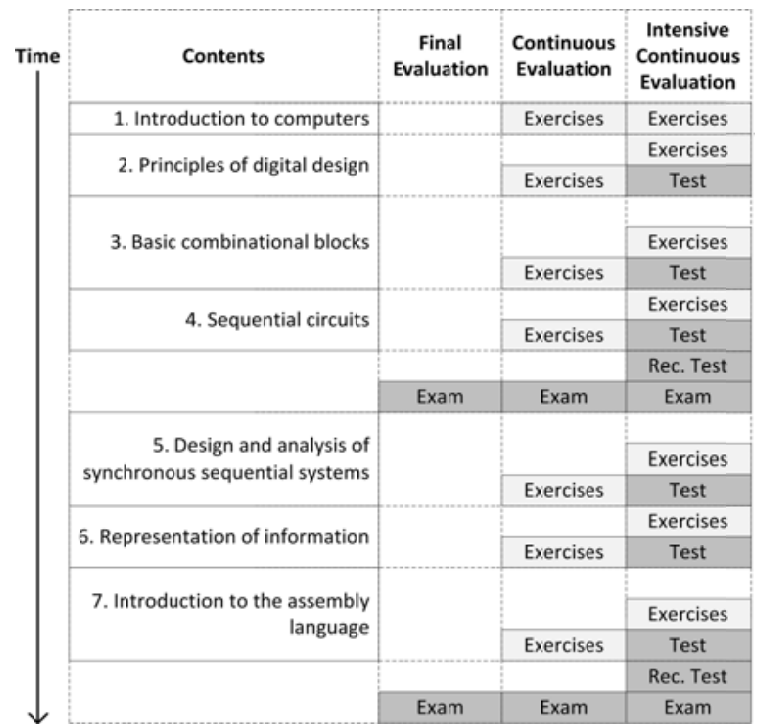

Fig. 2. Test schedule for the final evaluation methodology (left), continuous evaluation methodology (center) and the intensive continuous evaluation methodology (right), related to the various course units.

Fig. 2 shows when the continuous evaluation and final evaluation events take place, with respect to the course units covered. The second column shows the final evaluation (mandatory whichever type of continuous evaluation was adopted), which consists of a two-hour exam, containing about six or seven questions, that evaluates student understanding of the course contents after each half of the course. Note that these two final exams are common for all course sections, and take place not during class hours, but in a school-defined examination period.

In addition to these two evaluations, continuous evaluation, the third column, has students perform exercises associated with the various course units, with deliverables being evaluated by instructors and then returned to students.

Finally, intensive continuous evaluation, the far right column, includes an additional test for each course unit, to clearly determine students' level of competence for each unit.

All tests other than the final exams were performed in class, right after the last class of each unit; these are labeled as "Test" in Fig. 2. Since the aim of the tests for each unit was to measure knowledge acquisition during classroom sessions, and that students had virtually no time to prepare for the tests (which validated their level of understanding of the units, but not their learning level), two tests were given prior to the final examinations to allow students to recover from any bad marks obtained in previous tests. These "recovery" tests are labeled as "Rec. Test” in Fig. 2.

All intensive continuous evaluation tests were conducted as open book, with students being allowed to check their notes taken in class. This greatly facilitated students' acquisition of knowledge.

\section{EVALUATION OF THE PROPOSED METHODOLOGY}

As shown in the previous section, the implementation of the continuous evaluation and intense continuous evaluation strategies involved increasing the actual number of evaluation events, thus obviously increasing the instructor overhead. The main benefit of these strategies is that students gain significant feedback on their progress. Consequently, the evaluation results should improve compared to those of students receiving little or no feedback.

\section{A. Metrics}

To determine the overhead of continuous evaluation in terms of instructor effort, the associated overhead (represented as hours of activity) was measured for both the experimental group (suffix EG in the equations) and the control groups (suffix CG in the equations). The instructors' course-related activities were split into three categories: teaching, evaluation, and management. So, as shown in Equation 1, the overall instructor workload for the control group $\left(I w o_{C G}\right)$ is obtained from the sum of the time $(T)$, in hours, spent on these three academic activities: teaching $\left(T t_{C G}\right)$, evaluation $\left(T e_{C G}\right)$, and management $\left(\mathrm{Tm}_{\mathrm{CG}}\right)$

$I w o_{C G}=T t_{C G}+T e_{C G}+T m_{C G}$

For the experimental group, the additional load associated to the intensive continuous evaluation ( $\mathrm{Tec}$ ) must be added to Equation 1, resulting in Equation 2. Note that both the teaching load $\left(T t_{E G}\right)$ and the management load $\left(T m_{E G}\right)$ differ from the former case due to the extra management work involved in intensive continuous evaluation (several short meetings for coordination of new tests).

$I w o_{E G}=T t_{E G}+T e_{E G}+T e c_{E G}+T m_{C G}$

From the ratio between Equations 1 and 2 it is possible to obtain the instructor overload incurred when adopting intensive continuous evaluation, as shown in Equation 3:

$I O L=I w O_{E G} / I w O_{C G}$

To check the impact of intensive continuous evaluation on student results, the variable evaluation improvement $(E I)$ was defined (see Equation 4) as the ratio between the average student performance in the experimental group, and the average performance in the control group:

$E I=\overline{\operatorname{Perf}_{E G}} \overline{\operatorname{Perf}_{C G}}$

Based on the results of Equations 3 and 4, it is possible to obtain the normalized evaluation improvement (see Equation 5), which basically measures the student score increase as a function of the additional instructor load involved.

$$
\operatorname{Norm}(E I)=E I / I O L
$$

According to Equation 5, high values for Norm(EI) mean that the learning outcome is improved without excessive instructor effort, while low values mean that a lot of instructor 


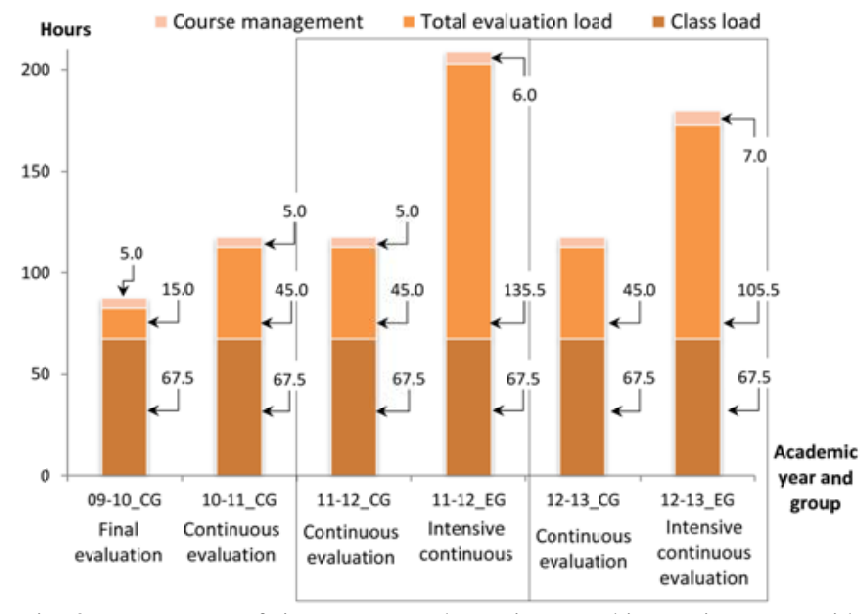

Fig. 3. Percentage of time spent on the various teaching assignments with respect to the type of assessment used.

effort results in just minimal gains in terms of student scores.

\section{B. Instructor Overload Results}

This section presents an analysis of the instructor overload associated with each evaluation strategy. Fig. 3 shows the percentage of time spent on each of the academic tasks related to the course for the different academic years analyzed. All instructors noted the time spent in every task on a spreadsheet. Collected times are displayed in Table II. As shown, the inclusion of continuous evaluation increases the evaluationrelated load from 5\% (15 out of 87.5 hours) to 38\% (45 out of 117.5 hours). However, when switching to intensive continuous evaluation, the load associated with evaluation tasks exceeds $50 \%$ of the total instructor effort on the course (135.5 out of 209 hours).

It is worth mentioning that, for an instructor, only class hours are recognized when measuring course load. Thus, depending on the type of evaluation made, the overall instructor load can vary significantly.

\section{Student Performance}

The previous section showed the remarkable increase of instructor load associated with new continuous evaluation teaching methodologies. This section examines whether this additional burden achieves significant improvements in terms of student performance.

For the performance analysis, two scores were compared: (i) the average score of the control groups on the final evaluation exam, and (ii) the average score of the experimental group on the final evaluation exam; note that intensive continuous evaluation was applied to the latter group (2011-12 and 2012-13 academic years).

The results presented in Fig. 4 show that the final scores of the control group are significantly better when continuous evaluation is used. This is an important, since it shows that the methodological changes introduced had a positive impact on student results, a conclusion consistent with previous studies

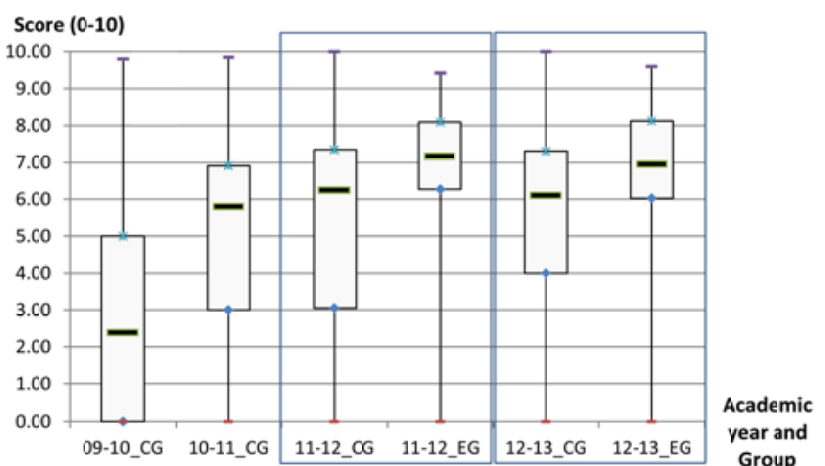

Fig. 4. Average student scores for the three types of evaluation adopted (CG=Control Group; EG=Experimental Group)

on this topic [15]. In the case of intensive continuous evaluation, the final evaluation results of the experimental group are better than the average score of the control groups. These results are based on the feedback provided by each of the course tests adopted for intensive continuous evaluation.

\section{Continuous Evaluation Results}

To conclude the analysis, this section summarizes the findings of this study using the metrics defined in Section IIIA. Table III shows the impact of continuous evaluation compared with final evaluation in terms of the proposed parameters. The Norm(EI) index shows that the extra effort invested by teachers (IOL of 34\%) implies an effective increase of the students' results (about $150 \%$ of $E I$ ). This result reinforces the arguments of the various studies presented in Section I, and justifies the adoption of continuous evaluation.

The most relevant results are shown in Table IV, which presents the results of student evaluation $(E I)$, instructor overload $(I O L)$ and normalized evaluation improvement $(E I)$ for the intensive continuous evaluation methodology for two consecutive academic years. These results highlight that the main instructor load associated with intensive continuous evaluation ranges between $77.9 \%$ and $53.2 \%$ of the total time dedicated to the course (management, classes and evaluation). That is, 90.5 additional hours during the 2011-12 academic year and 60.5 additional hours in the 2012-13 academic year compared to standard continuous evaluation. One of the reasons for this overload is the extra effort for continuous evaluation based on the additional recovery tests given to the experimental group (in addition to the exercises used in both experimental and control groups). Clearly, the main reason for the overload detected is the introduction of a test for each subject. Results for the EI parameter show that, indeed, these additional exams favor the improvements in student scores ( $14 \%$ on average). Nevertheless, it could be said that a new method is efficient only if the student score increase is proportional to the additional instructor overload. In terms of the Normalized EI parameter, this is achieved for values equal to or greater than 1 . In this study, the Normalized EI values obtained (0.19 and 0.26$)$ are too low to meet this criterion. 
TABLE II

DETAILED RESULTS OF INSTRUCTOR TIME (HOURS) SPENT ON THE COURSE/EVALUATION ELEMENTS

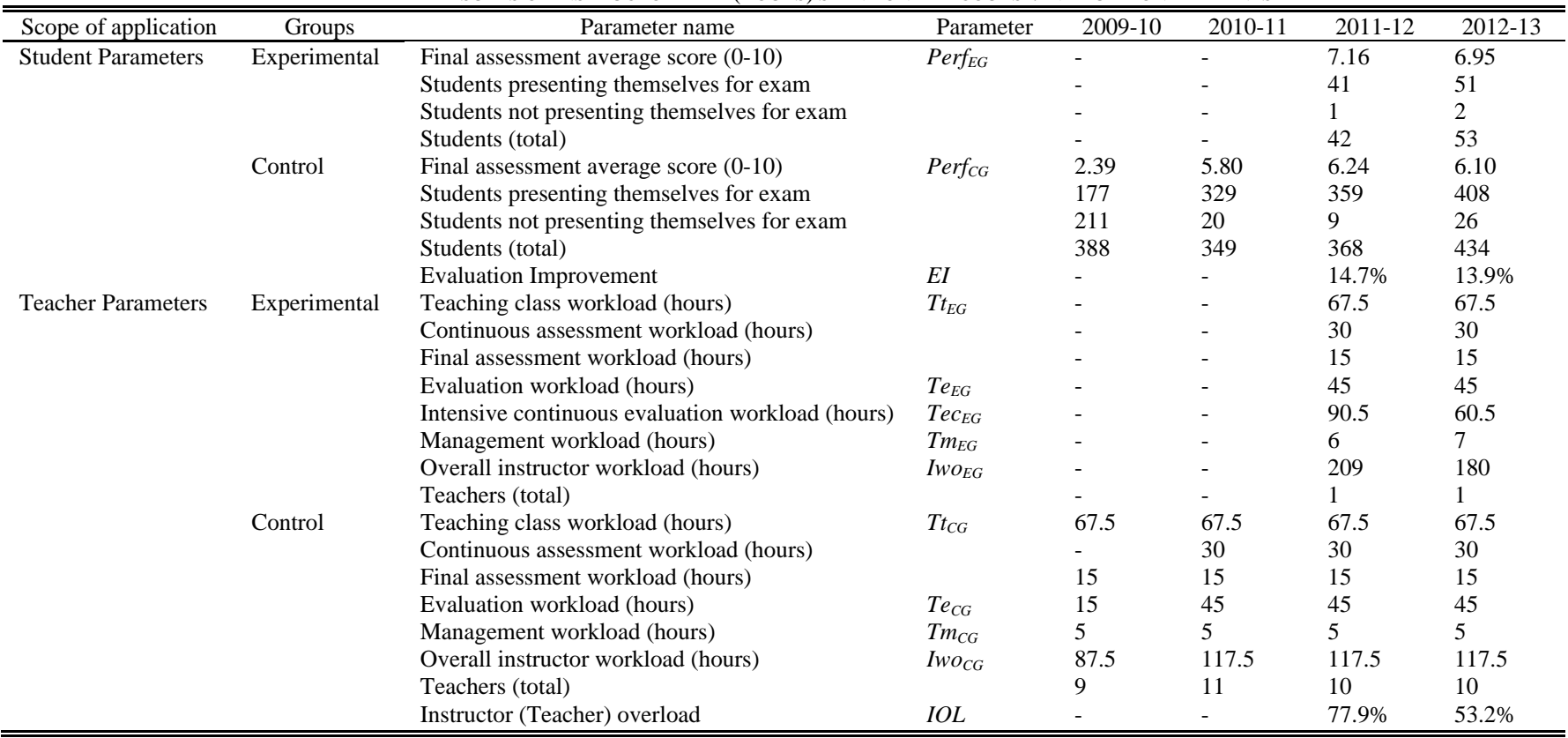

TABLE III

IMPACT OF CONTINUOUS EVALUATION IN TERMS OF THE PROPOSED PARAMETERS (CONTROL GROUPS ONLY)

\begin{tabular}{|c|c|c|c|c|}
\hline Parameter & $2009-10$ & $2010-11$ & $2011-12$ & $2012-13$ \\
\hline Evaluation type & Final & Continuous & Continuous & Continuous \\
\hline Perf $_{\mathrm{CG}}$ & 2.39 & 5.80 & 6.24 & 6.10 \\
\hline Iwo $_{\text {CG }}$ & 87.5 & 117.5 & 117.5 & 117.5 \\
\hline IOL Related to final & - & $34 \%$ & $34 \%$ & $34 \%$ \\
\hline $\begin{array}{l}\text { assessment } \\
\text { EI Related to final } \\
\text { assessment }\end{array}$ & - & $143 \%$ & $162 \%$ & $156 \%$ \\
\hline $\begin{array}{l}\text { Norm }(E I)_{\text {Related to }} \\
\text { final assessment }\end{array}$ & - & 4.18 & 4.71 & 4.54 \\
\hline
\end{tabular}

Overall, it is important to highlight that, although intensive continuous evaluation improved student scores, that improvement is not comparable to the additional effort these evaluation strategies imposed on the instructors. Alternatives must be sought that incur a lower instructor load.

\section{CONCLUSIONS}

Continuous assessment is one of the main pillars of the EHEA, being mandatory in new university degrees. Since it requires significant methodological changes, as well as its effectiveness, it is crucial to determine the actual burden this new system imposes on instructors.

Based on data obtained over several consecutive academic years, this paper has evaluated both the instructor effort associated with continuous evaluation, and student performance achieved using different evaluation methodologies: final evaluation, continuous evaluation (classroom exercises) and intensive continuous evaluation (classroom exercises plus one exam per unit). The results obtained yield significant quantitative conclusions:

1) Continuous evaluation and intensive continuous
TABLE IV

IMPACT OF INTENSIVE CONTINUOUS EVALUATION IN TERMS OF THE PROPOSED PARAMETERS (EXPERIMENTAL GROUP ONLY)

\begin{tabular}{lll}
\hline \hline \multicolumn{1}{c}{ Parameter } & \multicolumn{1}{c}{$2011-12$} & \multicolumn{1}{c}{$2012-13$} \\
& & \\
\hline Instructor overload (IOL) & $77.9 \%$ & $53.2 \%$ \\
Evaluation improvement (EI) & $14.7 \%$ & $13.9 \%$ \\
Normalized EI (Norm(EI)) & 0.19 & 0.26 \\
\hline \hline
\end{tabular}

evaluation improve student performance significantly, compared to final evaluation.

2) Intensive continuous evaluation also improves student results compared to continuous evaluation, but not so significantly.

3) The use of continuous evaluation and intensive continuous evaluation cause an increase of the teacher workload compared to final evaluation.

4) Intensive continuous evaluation increases the teacher workload very significantly compared to continuous evaluation.

The study shows that intensive continuous evaluation involves increased commitment and, consequently, more instructor effort. This increase may even imply that the time devoted by instructors to evaluation tasks may exceed $75 \%$ of the overall time devoted to the subject if intensive continuous evaluation methods are adopted. The use of continuous evaluation also affects student performance, which increased slightly. The current study found that this score increase can be, on average, up to $150 \%$. Therefore, it can be said that continuous evaluation improves student grades. However, increasing the number of tests and examinations (intensive continuous evaluation) is liable to create an excessive instructor overload without significantly increasing student 
scores (about 15\%).

In this respect, strategies such as online assessment, peer assessment, or self-assessment emerge as good candidate solutions, for a better trade-off between student performance and instructor overload.

As future work, some qualitative tests can complement the study presented here. For example, personal tests to gather student opinions of the feedback received from the exercises (continuous evaluation) and the topics of the written tests (intensive continuous evaluation), or even a parallel study to determine the effort (in hours) made by students, similarly to the instructor-oriented study presented in this paper, are worth tackling. It would also be interesting to reproduce this study in other courses to determine the influence of course contents on the various evaluation methods analyzed.

\section{REFERENCES}

[1] A.J. Lopez-Martin, "Attracting Prospective Engineering Students in the Emerging European Space for Higher Education" IEEE Transactions on Education, vol.53, no.1, pp.46-52, Feb. 2010. doi 10.1109/TE.2009.2023083

[2] M. Llamas-Nistal, M. Caeiro, M. Castro, I. Plaza and E. Tovar, "Engineering education in Spain: One year with the Bologna process" in Proc. EDUCON, Berlin, Germany, 2013, pp.566-572. doi: 10.1109/EduCon.2013.6530162

[3] D. Lopez, J.R. Herrero, A. Pajuelo and A. Duran, "A proposal for continuous assessment at low cost" in Proc. FIE, Milwaukee, WI, 2007, pp. 10-13, doi: 10.1109/FIE.2007.4417840

[4] R. Hernández, "Does continuous assessment in higher education support student learning?" Higher Education Vol.64 no.4, pp.489-502, 2012

[5] K.Cox, B. W. Imrie and A. Miller, Student assessment in higher education: a handbook for assessing performance. Routledge, 2014

[6] M. Combrinck and M. Hatch, "Students' Experiences of a Continuous Assessment Approach at a Higher Education Institution" J Soc Sci vol.33, no.1, pp.81-89, 2012

[7] A. Christofourou, A. Yigit, M. Al-Ansary, F. Ali, A. Aly, H. Lababidi, I. Nashawi, A. Tayfun and M. Zribi, "Improving Engineering Education a Kuwait University through Continuous Assesment” International Journal Engineering Vol.19. no.6, pp.818-827, 2003

[8] Engineering Criteria 2000, 3 Ed., Accreditation Board for Engineering and Technology, Inc., Baltimore, MD, 1997

[9] M. Llamas-Nistal, "An Experience of Continuous Assessment in Telecommunication Technologies Engineering: New Costs for the Teacher." IEEE Revista Iberoamericana de Tecnologías del Aprendizaje, vol.8. no.2, pp.90-95, 2013, doi 10.1109/RITA.2013.2258225

[10] A. Sursock, H. Smidt and H. Davies. Trends 2010: A decade of change in European Higher Education, Ed. European University Association, Brussels, 2010

[11] G.S. Hanna and P.A. Dettmer, Assessment for effective teaching: Using context-adaptive planning, Boston, MA, Pearson A\&B, 2004

[12] W. Harlen and M. James, "Assessment and learning: differences and relationships between formative and summative assessment" Assessment in Education, Vol.4, no.3, pp. 365-379, 1997

[13] M. Scriven, "Beyond Formative and Summative Evaluation," in Evaluation and Education: A Quarter Century, M.W. McLaughlin and ED.C. Phillips, eds. Chicago: University of Chicago Press, 1991

[14] Normativa de Régimen Académico y Evaluación del Alumnado de la Universitat Politècnica de València. UPV 2010. Available: http://www.upv.es/upl/U0490393.pdf

[15] M. D. Cano, "Students' Involvement in Continuous Assessment Methodologies: A Case Study for a Distributed Information Systems Course," IEEE Transactions on Education, vol.54, no.3, pp.442-451, Aug. 2011.
Jose-Luis Poza-Lujan (M’12) Valencia, Spain 1969, received the B.S. degree in education from Valencia University in 1990 and the B.S. degree in computing from Technical Valencia University, Valencia (UPV), in 1999. He received his Ph.D. degree in informatics from the Technical University of Valencia in 2012, where he has worked since 2000. He is a currently a lecturer and researcher at the UPV, in the fields of smart devices and intelligent distributed control networks. His educational research interests include engineering soft skills and students' competency management.

Carlos T. Calafate is an associate professor in the Department of Computer Engineering at the Technical University of Valencia (UPV) in Spain. He graduated with honors in electrical and computer engineering at the University of Oporto (Portugal) in 2001. He received his Ph.D. degree in informatics from the Technical University of Valencia in 2006, where he has worked since 2002. His research interests include ad-hoc and vehicular networks, mobile applications, QoS, network protocols, video streaming, and network security.

Juan-Luis Posadas-Yagüe received the M.Sc. and Ph.D. degrees in computer engineering from the Universitat Politècnica de València (UPV), Spain, in 1996 and 2003 respectively. He is currently an associate professor in the Department of Computer Engineering at the UPV, and has taught computer architecture in the School of Engineering in Computer Science since 1997. His research interests include distributed systems, real-time mobile robot control and coordination, code delegation, multi-agent systems and embedded systems.

Juan-Carlos Cano is a full professor in the Department of Computer Engineering at the Polytechnic University of Valencia (UPV) in Spain. He earned an M.Sc. and a Ph.D. in computer science from the UPV in 1994 and 2002 respectively. From 1995-1997 he worked as a programming analyst at IBM's manufacturing division in Valencia. His current research interests include vehicular networks, mobile ad hoc networks, and pervasive computing. 